\title{
VARIACIÓN GENÉRICA DE LAS JUSTIFICACIONES ATENUANTES EN ESPAÑOL CHILENO ${ }^{1}$ \\ GENERIC VARIATION OF MITIGATING JUSTIFICATIONS IN CHILEAN SPANISH
}

\author{
Silvana Guerrero González \\ Universidad de Chile \\ siguerrero@u.uchile.cl \\ Consuelo Gajardo Moller \\ Pontificia Universidad Católica de Chile \\ cgajardom@uc.cl \\ Daniela Ibarra Herrera \\ Pontificia Universidad Católica de Chile \\ dvibarra@uc.cl \\ Javier González Riffo \\ Universidad Católica Silva Henríquez \\ jgonzalezr@ucsh.cl \\ Antonia Reyes O’Ryan \\ Universidad de Chile \\ antonia.reyes.o@ug.uchile.cl
}

\begin{abstract}
RESUMEN
En esta investigación se presentan los resultados más relevantes sobre el análisis de las justificaciones atenuantes en dos géneros discursivos: la conversación coloquial y la entrevista semidirigida. Específicamente, se estudian las justificaciones en los materiales de los corpus AMERESCO y PRESEEA de Santiago de Chile, a partir de cinco dimensiones: lingüística, pragmático-discusiva, estructural, enunciativa y situacional. Los principales hallazgos del estudio sugieren que, si bien existen similitudes entre ambos géneros revisados, las estrategias de atenuación para justificar se utilizan de formas muy típicas en un género determinado en función del tipo de registro. Se destacan los factores
\end{abstract}

\footnotetext{
${ }^{1}$ Este trabajo se enmarca en el proyecto de investigación "La atenuación lingüística en el español chileno: enfoque pragmalingüístico y sociopragmático" (ANID/CONICYT Fondecyt Regular 1200003).
} 
situacionales relacionados con la diversidad temática o la relación de igualdad entre los hablantes como determinantes en el funcionamiento de las justificaciones atenuantes.

Palabras clave: atenuación, justificaciones, imagen, PRESEEA, AMERESCO.

\begin{abstract}
This investigation presents the most relevant results regarding the analysis of mitigating justifications in two discursive genres: colloquial conversation and semi-structured interview. Specifically, the justifications are studied in corpora of AMERESCO and PRESEEA from Santiago, Chile, considering five dimensions: linguistic, pragmaticdiscursive, structural, enunciative and situational. The study's main findings suggest that, even though there are similarities between the two genres studied, the mitigating strategies used to justify are used in rather characteristic ways in a determined genre, according to the type of register. Some situational factors are brought into focus, such as the thematic diversity or the equal relationship between speakers, which are regarded as key elements in the functioning of mitigating justifications.
\end{abstract}

Key words: mitigation, justifications, face, PRESEEA, AMERESCO.

Recibido: 01-09-20

Aceptado: 06-11-20

\title{
1. Introducción
}

Las investigaciones en torno al fenómeno de la atenuación lingüística en el castellano chileno han aumentado en los últimos años (Puga; Montecino Cortesía y modalización, Intensificadores en el habla; González Riffo y Guerrero González Estudio sociolingüístico, Estrategias de atenuación, Recursos de atenuación; González Riffo Estrategias de atenuación, Diminutivos atenuadores; Guerrero y Guerrero et al.). Sin embargo, estos trabajos se han centrado en la descripción pragmática del fenómeno o en su distribución sociolingüística. Como se verá a continuación, en otras variedades del español ha habido en el último tiempo un fuerte interés por el estudio de la atenuación en su variación genérica. El resultado del trabajo científico ha dado origen, por ejemplo, a números monográficos de revistas especializadas, como el editado por Albelda Estrategias atenuantes. Entre las diversas investigaciones que se han centrado en el género discursivo en el que se emplea la 
atenuación, destacan la de Bouzas, quien trabaja con corpus proveniente de la prensa; la de Moya y Carrió-Pastor, los cuales trabajan sobre la base de comentarios de prensa on line; las de Estrada y Zárate e Infante y Flores, que abordan el estudio de la atenuación en el discurso político; las de Contreras, González y García, Helfrich y Pano sobre la atenuación en diversos medios de comunicación electrónicos, y la de De Cock et al. en que se comparan los atenuantes en los géneros conversación coloquial, entrevista semidirigida, interacción en clases y debate parlamentario.

Esta investigación se propone llevar a cabo una descripción de la atenuación en dos géneros discursivos: la conversación coloquial y la entrevista semidirigida, centrando el análisis en las justificaciones. Considerando que una diferencia capital entre ambos géneros corresponde a los roles que desempeñan los hablantes en la interacción, lo que se relaciona con el registro +/- formal o +/- coloquial, este trabajo supone conocer el contexto interactivo donde se desarrolla la atenuación (Meyer-Hermann; Villalba). Hasta donde tenemos conocimiento, esta investigación es pionera en el estudio de la variedad chilena del español.

Los estudios empíricos han demostrado que una de las estrategias de atenuación más frecuentes en la variedad de habla chilena son las justificaciones (Guerrero). Estas tienen por función reducir el compromiso epistémico del hablante hacia la verdad que está expresando o suavizar o relativizar la expresión de los juicios u opiniones del propio hablante, quien calcula que su imagen podría verse afectada (Albelda ¿Cómo se reconoce). Considérese el siguiente ejemplo de uso:

(1) A: ¿no lo leíste o te hiciste el huevón ${ }^{2}$ ?

B: $o$ sea no sé pero te acordái quee-

$\mathrm{C}:[((\quad))$ ¡ah pero ustedes estaban en el a!]

$\mathrm{B}$ : [es que me sentaba con el C y] el P y [cada/ cada/ cada]

C: [¿vos estábai en el b?] (AMERESCO_SCL_001_003_18) ${ }^{3}$

\footnotetext{
2 "Huevón" en español de Chile se refiere a un término coloquial comúnmente utilizado como vocativo entre amigos y también puede usarse con significado denostativo. Además de adjetivo, se puede usar como verbo "hueviar" y como sustantivo "huevada".

3 Los ejemplos del corpus AMERESCO se transcriben ortográficamente, conservando los elementos esenciales del sistema de transcripción. Los nombres propios se reemplazan por letras mayúsculas. Al final de cada ejemplo se codifica, entre paréntesis, la información relativa a la conversación: SCL $=$ Santiago de Chile; el número que sigue es el número de la grabación en el corpus; luego se hace referencia a la cantidad de informantes por conversación y, finalmente, se hace mención al año de la grabación. Se usa cursiva y negrita para identificar los atenuantes aludidos, y solo cursiva para marcar el resto de los atenuantes.
} 
El ejemplo precedente ilustra el funcionamiento de la justificación atenuante en un corpus conversacional. Un grupo de amigos comenta el libro "La Peste", lectura que, a pesar de ser obligatoria en la escuela secundaria, uno de ellos (B) no leyó. La pregunta desaprobatoria de A (“¿no te lo leíste o te hiciste el huevón?”) actúa como desencadenante de la justificación de $\mathrm{B}$, quien excusa su comportamiento atribuyéndole la responsabilidad a terceros ("es que me sentaba con el $C$ y el $P$ "). La justificación funciona como estrategia para reducir el compromiso del hablante frente a la acción que se le atribuye, esto es, no leer un clásico obligatorio, y también para suavizar los potenciales juicios de sus interlocutores, lo que es calculado como un riesgo para su imagen y, en consecuencia, también para los fines interpersonales de la conversación. Pese a que en la mayoría de las ocasiones se puede observar este menor compromiso del hablante, también puede darse el caso de que cuando se justifica algo no ha habido un compromiso previo del hablante hacia ninguna verdad o certeza, pues el hablante solo da razones para que no se le atribuya un juicio que sospecha que se le puede atribuir.

En el marco de la pragmalingüística, esta investigación se propone específicamente: (a) caracterizar las funciones de imagen que cumplen las justificaciones atenuantes en los géneros en estudio; (b) establecer los tipos de actos de habla, su fuerza ilocutiva y los tipos textuales atenuados en cada género discursivo; y, (c) comparar el funcionamiento de las estrategias para justificar empleadas en los dos tipos de registros $+/$ - coloquial y $+/$ - formal, según los géneros revisados. Para cumplir estos objetivos, se analizan los materiales correspondientes al español chileno provenientes de dos proyectos: el proyecto AMERESCO (América y España Español Coloquial) ${ }^{4}$, destinado a recoger muestras de conversaciones coloquiales espontáneas de diferentes áreas de América y España, y el proyecto PRESEEA (Proyecto para el Estudio Sociolingüístico del Español de España y América), que trabaja con base en entrevistas sociolingüísticas semidirigidas.

Con el propósito de ampliar lo que ya se sabe de la atenuación en el español chileno, en esta investigación se describen las justificaciones atenuantes en cinco dimensiones:

\footnotetext{
${ }^{4}$ Este proyecto se origina en el contexto de los proyectos Es.Var.Atenuación (La atenuación pragmática en el español hablado: su variación diafásica y diatópica) y Es.Vag.Atenuación (La atenuación pragmática en su variación genérica: géneros discursivos escritos y orales en el español de España y América) en la Universidad de Valencia. En todos los casos se sigue la metodología del corpus Val.Es.Co. (Briz y Val.Es.Co.).
} 
lingüística, pragmático-discusiva, estructural, enunciativa y situacional. Se trata de un estudio cualitativo, que se constituye como un acercamiento al análisis de las estrategias de atenuación como fenómeno variable en el habla de Chile, en un corpus oral y actual. Este estudio sobre la justificación atenuante intenta contribuir, además, a las investigaciones que se han enfocado en fenómenos discursivos a partir de los presupuestos teóricos de la pragmalingüística, de modo que permite indagar en la disponibilidad de recursos que posee un hablante para participar en actos comunicativos y construir significados relacionales e interpersonales.

\section{Marco teórico}

\subsection{Atenuación lingüística e imagen}

Si bien Briz El español coloquial constituye una lectura obligatoria para el estudio de la atenuación, en esta investigación se ha preferido trabajar sobre la base de una definición más unificadora y actualizada de lo que se entiende por atenuación:

Estrategia retórico-pragmática originada por necesidades de protección de la imagen (propia o ajena), para proteger, suavizar y reparar los posibles efectos perjudiciales para el adecuado desarrollo de la comunicación. Se expresa a través de mecanismos de lenguaje vago que difuminan el contenido proposicional, de minimización de la cantidad o cualidad semánticas o directamente reduciendo la fuerza ilocutiva de los actos de habla y formulando un menor compromiso con lo dicho. Genera una implicatura conversacional a través de la indireccionalidad en la expresión de la verdadera intención del emisor (Albelda y Cestero Estudio de variación).

Como proponen Briz y Albelda y Albelda Sobre la incidencia, la atenuación linguiística funciona como una categoría pragmática (mecanismo estratégico, dirigido a la efectividad y eficacia discursiva), una estrategia (que atenúa argumentativamente, para lograr el acuerdo o la aceptación del otro) y un mecanismo retórico (para convencer, persuadir y cuidar las 
relaciones sociales e interpersonales o evitar su menoscabo), de manera que se vincula con la noción de Goffman de imagen - puesto que los individuos están comprometidos con una imagen de sí mismos-, y por lo tanto con las reglas para el contacto interpersonal. Así, la imagen constituye el sentido reclamado por la persona de valoración social favorable del yo en un contexto relacional (Figueras); en este sentido, de acuerdo con la autora, opera como un recurso de carácter identitario vulnerable, que puede amenazarse o realzarse en cualquier situación social.

\subsection{Justificación atenuante}

Briz El español coloquial alude a los rodeos justificativos para referirse a aquellos actos de habla que emplean estrategias atenuantes como "es que" para suavizar su fuerza ilocutiva. Albelda y Cestero De nuevo denominan a este grupo de actos como justificaciones o excusas, y con ello hacen alusión a un movimiento cognitivo y comunicativo que incluya cualquier mecanismo que apoye argumentativamente lo dicho y suponga, a la vez, una reducción del peso enunciativo del hablante. Las autoras precisan que las justificaciones en tanto estrategia atenuante pueden concretarse mediante justificaciones y excusas de lo dicho o del decir, a través del empleo de una comparación como forma de justificarse y empleando el uso de marcadores discursivos que presentan consecución lógica.

Un trabajo pionero sobre las justificaciones en actos de habla asertivos es el que desarrollan

Flores y Ramírez acerca del español de Monterrey. Estos autores clasifican en cuatro subtipos las justificaciones atenuantes: justificaciones, explicaciones o excusas, comparaciones y marcadores discursivos que desempeñan la función de conectores lógicos. Sobre la base de esta investigación, Guerrero et al. analizan estos recursos en el español chileno y proponen tres estrategias asociadas con la idea de justificar los actos de habla asertivos: (a) justificaciones, a través de las cuales el hablante ofrece razones que considera convincentes para probar algo; (b) explicaciones, mediante las que el hablante da a conocer, declara o manifiesta la causa o motivo de algo, aclarando lo dicho; y, (c) comparaciones, en cuyo caso el hablante fija la atención en dos o más objetos o situaciones para mostrar sus relaciones, diferencias o semejanzas. Estos autores señalan que las justificaciones se 
configuran mediante una conclusión y (negación) porque / que / lo que pasa es (que) / es que / ya que (+ marcador discursivo) $x 1, x 2, x \mathrm{n}$ argumentos. Las explicaciones, por su parte, presentan primero los argumentos $x 1, x 2$, xn por eso (que) conclusión $y$, y, finalmente, las comparaciones adoptan una forma donde $x=y$.

\subsection{Factores determinantes}

A continuación, se presentan las cinco dimensiones teórico-analíticas que se incluyen en esta investigación, siguiendo la propuesta original de Albelda y Cestero De nuevo, Cestero y Albelda et al.

a) Factores lingüísticos: estrategias lingüísticas para justificar

En este trabajo se incluyen las tres estrategias específicas para justificar actos de habla asertivos ya estudiadas en el español chileno: justificaciones, explicaciones y comparaciones (Guerrero et al.).

b) Factores pragmático-discursivos: funciones de la atenuación

Se consideran dos factores: 1) el tipo de acto de habla que incluye discurso atenuado según su fuerza ilocutiva y 2) las funciones básicas de la atenuación: a) autoprotección (salvaguarda del yo), esto es, el uso de atenuadores para evitar o reducir el compromiso del hablante con lo dicho o su repercusión y para salvar la imagen propia; b) prevención (salvaguarda del yo y del tú), en cuyo caso se tiende a evitar las repercusiones de lo dicho o hecho e imponer la opinión propia, buscando acuerdo o justificando el desacuerdo; se previenen posibles daños a la imagen o problemas por invadir el territorio del otro; y, c) reparación, cuya función es mitigar o reparar una amenaza al interlocutor.

c) Factores estructurales

Se incluye aquí el estudio de la tipología textual en que aparece el atenuante; esto es, se discrimina las secuencias según tipologías narrativas, descriptivas y argumentativas. Asimismo, se toma en consideración el o los lugares, en términos de posición, de la actividad retórica donde aparece la estrategia atenuadora.

d) Factores enunciativos 
En este caso se atiende a los elementos que definen al acto de habla como tal, por lo tanto, queda incorporado el análisis de la fuerza ilocutiva del acto de habla.

e) Factores situacionales

Se incluye el estudio de dos tipos de factores: 1) el tipo de registro +/- formal y +/coloquial, según los géneros revisados y 2) la temática.

\section{Metodología ${ }^{5}$}

\subsection{Corpus e informantes}

En esta investigación se incluye la revisión de materiales proveniente de dos corpus de español chileno. Primero, se analiza el corpus correspondiente al proyecto AMERESCO de Santiago de Chile (Guerrero González y González Riffo). Puesto que se trata de una primera aproximación a la variación genérica de las justificaciones atenuantes, se analizan 27 conversaciones coloquiales y espontáneas grabadas. En dichas conversaciones los hablantes interactúan con otros -entre 2 y 3 personas por conversación- con quienes mantienen una relación de alta proximidad, un marco de interacción cotidiano y una cotidianidad temática. Cada grabación tiene una duración de 20 minutos. En segundo lugar, se estudia el corpus PRESEEA de Santiago de Chile de entrevistas semidirigidas (PRESEEA), en cuyo caso un entrevistador realiza una entrevista a un informante con quien no necesariamente mantiene una relación de alta proximidad, un marco de interacción cotidiano y una cotidianidad temática. Tomando como base la Guía de Estudios de la Atenuación en los corpus PRESEEA ${ }^{6}$, se seleccionan 30 minutos de grabación (minutos 10 al 40) de cada entrevista. Consecuentemente, en cada corpus se analizan 9 horas de registro de discurso natural.

\footnotetext{
${ }^{5}$ Esta metodología reproduce, en gran medida, la que se utiliza para todas las investigaciones que se realizan en el Fondecyt Regular 1200003.

${ }^{6}$ Documento disponible en la página Web del proyecto PRESEEA http://preseea.linguas.net/ sección Metodología.
} 
Para ilustrar el funcionamiento de los rasgos propios de los géneros discursivos que se analizan en este trabajo, se expone la tabla 1. Cabe precisar que mientras las conversaciones coloquiales corresponden a un género que se sitúa en el eje de coloquialidad, las entrevistas semidirigidas, por su parte, son tendientes al eje de formalidad.

Tabla 1. Escalas de variación situacional (simplificada de Briz El español coloquial)

\begin{tabular}{|c|c|c|c|}
\hline \multicolumn{2}{|c|}{$\begin{array}{l}\text { EJE DE LA COLOQUIALIDAD } \\
\text { COMUNICACIÓN COLOQUIAL } \\
\text { + INMEDIATEZ COMUNICATIVA }\end{array}$} & \multicolumn{2}{|c|}{$\begin{array}{r}\text { EJE DE LA FORMALIDAD } \\
\text { COMUNICACIÓN FORMAL } \\
\text { - INMEDIATEZ COMUNICATIVA }\end{array}$} \\
\hline + coloquial prototípico & - coloquial periférico & - formal periférico & \begin{tabular}{l|l} 
& + formal prototípico \\
\end{tabular} \\
\hline \multicolumn{2}{|c|}{ RASGOS PROPIOS DEL REGISTRO COLOQUIAL } & \multicolumn{2}{|c|}{ RASGOS PROPIOS DEL REGISTRO FORMAL } \\
\hline \multicolumn{2}{|c|}{+ planificación sobre la marcha + +/- } & \multicolumn{2}{|c|}{$+/-\quad$ - planificación sobre la marcha } \\
\hline \multicolumn{2}{|c|}{+ fin interpersonal } & $+/-$ & - fin interpersonal \\
\hline \multicolumn{2}{|l|}{+ tono informal } & $+/-$ & - Tono informal \\
\hline
\end{tabular}

Por último, debe aclararse que, si bien ambos corpus están estratificados por sexo, edad y nivel de instrucción, para efectos de este estudio las variables sociales no fueron consideradas.

\subsection{Procedimientos analíticos}

Para el análisis de los datos se utilizó como unidad analítica el atenuante en un contexto interaccional concreto (Briz y Albelda). De esta forma, se incluyen los siguientes tres segmentos: 1) el miembro del discurso afectado por la atenuación, 2) el miembro del discurso causante o desencadenante y 3) la expresión atenuante concreta. En paralelo, se adoptó la metodología de análisis propuesta por Cestero y Albelda, Briz y Albelda y Albelda et al. para identificar la estrategia de justificar con función atenuante.

Para llevar a cabo este estudio se consideró como supuesto cardinal que la atenuación mediante justificaciones en los actos asertivos ocurre cuando está claramente comprometida alguna de las imágenes de los interlocutores. Para el investigador, entonces, debe resultar sencillo identificar si el mensaje transmitido supone una carga negativa (Albelda ¿Cómo se reconoce). Por consiguiente, se siguieron cuatro pasos fundamentales: (a) se identificaron todos los casos donde se justificaba para atenuar en los géneros en estudio; (b) a nivel pragmático-discursivo, se revisaron las funciones de imagen que cumplían las justificaciones en ambos géneros; (c) en la dimensión estructural, se tomó en consideración 
la tipología textual -narrativa, descriptiva o argumentativa- donde se incluían las justificaciones; (d) en la dimensión enunciativa se estudió el acto de habla atenuado; y, (e) para completar el análisis situacional, se revisó el tipo de registro +/- formal y +/- coloquial, y la temática asociada con el empleo del atenuante.

Por último, se trata de un análisis inductivo ${ }^{7}$ que trabajó con tres pruebas: ausencia, conmutación y solidaridad (Villalba 312 y ss.):

1) Prueba de la ausencia: consiste en eliminar el elemento atenuante y observar si se producen cambios en la fuerza ilocutiva.

2) Prueba de la conmutación: si no se puede aplicar la prueba (a), se sugiere intentar sustituir el atenuante por otras opciones que desempeñen la misma función. Si se lo sustituye por una forma más directa y se produce un incremento en la fuerza ilocutiva, el segmento será un atenuante.

3) Prueba de la solidaridad: puesto que la atenuación no suele aparecer de manera aislada, debiese haber presencia de más mecanismos atenuantes en el segmento que se está analizando.

En este trabajo hemos optado por usar estas tres pruebas de manera complementaria en la tarea de verificar el carácter atenuante de las expresiones revisadas.

\section{Presentación y discusión de resultados}

Para completar los objetivos propuestos en esta investigación, se exponen contrastivamente los principales resultados del análisis empírico ejecutado. Debe precisarse que los hallazgos dicen relación con la diferencia entre lo que se entiende por rasgos coloquializadores (+ relación de igualdad, + relación vivencial, + marco de interacción cotidiano y + cotidianidad temática) y rasgos de formalidad (- relación de igualdad, - relación vivencial, marco de interacción cotidiano y cotidianidad temática) (Briz El español coloquial). En este sentido, era de esperar que hubiese gran preponderancia de justificaciones atenuantes en la entrevista semidirigida, en comparación a la conversación coloquial, dado que presentan un mayor control de lo producido (Briz y Albelda).

\footnotetext{
${ }^{7}$ No está demás advertir que en esta investigación no se ofrecen datos cuantitativos, sino solo cualitativos.
} 


\subsection{Sobre los factores lingüísticos: estrategias lingüísticas para justificar}

Las estrategias lingüísticas que se emplean para atenuar las justificaciones en la conversación coloquial son las justificaciones, las explicaciones y las comparaciones, en el sentido que sugieren Guerrero et al. A este respecto, debe aclararse que en este estudio el término ‘justificaciones' opera a la vez como hiperónimo e hipónimo, de modo que lo que se analiza corresponde a los tres "subtipos" de justificaciones. Asimismo, es interesante que la justificación es en sí el segmento lingüístico (o el acto de habla) que constituye una atenuación, esto es, la justificación atenúa a otro segmento u otro acto de habla (como ocurre en el ejemplo (1)), o también puede atenuar una visión, percepción u opinión que se tiene de uno de los hablantes o de un tema (como ocurre en el ejemplo (4)). Esta matización es esencial, puesto que hay otras estrategias atenuantes que no constituyen un acto de habla completo en sí, y que por lo mismo no podrían ser autónomas per se, como sí lo es una justificación. Además, dentro del acto de justificación, podrían emplearse también otras estrategias atenuantes, como diminutivos, verbos de opinión, formas vagas, impersonalizaciones, etc., para atenuar todavía más, lo que de por sí constituye un acto de habla atenuado.

En el género conversación coloquial, la estrategia lingüística mayormente utilizada es la justificación propiamente tal en actos de habla asertivos, seguido por la explicación y, en menor medida, las comparaciones. Como se trata de un recurso muy productivo en este género, las justificaciones muestran variadas combinaciones y herramientas lingüísticas, entre las que destacan: (marcador discursivo pero o claro +) es que, es que (+ marcador discursivo), lo que pasa es que, distintas combinaciones de sustantivo + es que (como el cuento es que, la huevada es que, la cosa es que, el tema es que, el problema es que, la cuestión es que), que, no es que [...] es que, porque + es que, no es que [...] pero, no es por $[\ldots]$ es porque, porque e incluso se encuentran combinaciones de hasta tres de estos recursos consecutivos en un mismo turno de conversación, como muestra el siguiente ejemplo: 
(2) A: [(((J también era mal profe $)))]$

$\mathrm{C}$ : [a mí igual $\mathrm{C}$ no me caía tan bien huevón]

B: sí y- y no aprendí nada yo creo huevón solo leí un par de libros así

$\mathrm{C}:[($ noo $\mathrm{C}$ era una mierda en ese sentido)]

A: [de- de- desde lo que es contenido-] desde lo que es el contenido el huevón era un muy mal profe

$\mathrm{C}: \mathrm{C}$ era un [troll $^{8}$ culeado $^{9}$ ]

B: [(( )) sí huevón]

A: es que-lo que pasa es que-el problema es que esos huevones simplifican demasiado la huevada po huevón (AMERESCO_SCL_001_03_18).

En este ejemplo, tres amigos (A, B y C) critican a quienes fueron sus profesores de colegio. En el juicio general parece no haber controversia con respecto a la percepción negativa que tienen sobre sus profesores. Sin embargo, sí parece haber diferencias con respecto a las razones que fundamentan estas creencias: mientras A señala que su percepción se origina por la calidad de las clases del docente ("desde lo que es contenido el huevón era un muy mal profesor"), В y C responden enjuiciando las habilidades blandas de este ("era un troll culeado"). Este desencuentro podría constituir una amenaza para la imagen de A, por lo que sirve de desencadenante de la justificación que este interlocutor realiza posteriormente, introducida a través de es que. Es importante señalar que la naturaleza informal y menos planificada de la conversación coloquial permite reformulaciones y, por lo tanto, estas secuencias de más de dos recursos lingüísticos de justificación atenuante.

Como se señaló anteriormente, la explicación muestra una menor fundamentación que las justificaciones y se registra en el género conversación coloquial únicamente en las formas (marcador discursivo +) por eso, no por eso y por eso. Finalmente, las comparaciones se realizan a través de es como y como que. En este sentido, es relevante precisar que la mayor parte de los ejemplos, salvo que se indique otra cosa, corresponde al subtipo justificación.

\footnotetext{
8 "Trolero", a, del inglés troll: expresión juvenil utilizada para referirse a una situación cuyo humor es particular. Puede ser sustantivo o adjetivo, además de poseer forma verbal (trolear).

9 "Culeado" es el participio de "culear". Generalmente se utiliza como sinónimo de "desgraciado" o "huevón".
} 
En el género entrevista semidirigida también se registran justificaciones propiamente tal, explicaciones y comparaciones. Mayoritariamente se emplean justificaciones de los actos de habla asertivos introducidos a través de las siguientes combinaciones de recursos lingüísticos: lo que pasa es que (+ marcador discursivo), es que (+ marcador discursivo), porque (+ marcador discursivo), no es porque [...] pero, no es porque [...] sino que у es que encuentro que. Las explicaciones en este género se construyen mediante por eso, por eso que, así que (+ marcador discursivo) y ya que. Por último, las comparaciones se introducen por medio de es como y es como que. Si bien en general se utiliza una sola estrategia linguística para justificar los actos de habla asertivos, hay casos en los que los hablantes hacen uso de justificaciones, explicaciones y comparaciones en un mismo segmento discursivo con el propósito de atenuar aquello que afirman. Esto ocurre en mucha menor medida en el corpus conversacional, probablemente porque los turnos de conversación son más dinámicos y significativamente menos extensos. Considérese, en contraste, el siguiente ejemplo del corpus PRESEEA de entrevista semidirigidas:

(3) E: ya y y ¿qué? // bueno y ¿qué hacen generalmente cuando van / van a la playa / van a comprar? // I: sí / lo que pasa es que allá / Loncura es como es como un pueblito así como / es bien piola / y y fome por lo demás / así que hay que ir a Quintero / en Quintero están los juegos / está la plaza y uno a ver a la gente ahí a los artistas ¿cachái? / a comprar las cosas / porque allá hay como dos almacenes y es fome / pero lo bueno que allá la playa está cerca / y en la noche es rico bajar a la playa y fumarte un cigarro o otra cosa no sé / pero como ando con mis padres no puedo / pero es rico estar ahí en la noche mirando el mar es rico eso $\left(\right.$ PRESEEA_SCHI_M12_043) ${ }^{10}$.

\footnotetext{
${ }^{10}$ Los ejemplos del corpus PRESEEA se transcriben de manera ortográfica y al final de cada uno se codifica, entre paréntesis, la información relativa al informante. Debe considerarse que $\mathrm{E}=$ entrevistado e $\mathrm{I}=$ informante; $\mathrm{SCHI}=$ Santiago de Chile; $\mathrm{H}=$ Hombre y $\mathrm{M}=$ Mujer. El primer número, luego del sexo del informante, corresponde al grupo etario: $1=20-34$ años; $2=35-54$ años y $3=55$ años y más. El segundo número corresponde al nivel educacional del informante: 1 = estudios básicos completos o incompletos; $2=$ estudios medios completos o incompletos y 3 = estudios universitarios completos o incompletos. El número que sigue al guion corresponde al número correlativo que reciben los sujetos que conforman la muestra. Solo se analizan los actos de habla del informante: se usa cursiva y negrita para identificar los atenuantes aludidos, y solo cursiva para marcar el resto de los atenuantes.
} 
En este caso, la informante, frente a la pregunta que opera como desencadenante del acto de habla, comienza justificándose, porque considera el lugar de vacaciones poco atractivo y probablemente asume que también lo sería para su entrevistador, por lo tanto, su intervención busca no solo autoprotegerse, sino que también prevenir un conflicto con el tú al que se dirige. Sobre la comparación, el informante le atribuye a "Loncura" las cualidades de un "pueblito" asumiendo que su interlocutor podría conocer otras localidades de características comparables. Un desencadenante como el citado debería haber generado una descripción y, sin embargo, el discurso se articula como una argumentación que permite explicar finalmente por qué deben trasladarse de una playa a otra para realizar las compras. De hecho, la informante destaca hacia el final de su intervención que también hay algo positivo en el lugar donde vacaciona ("pero lo bueno que allá la playa está cerca”); de este modo, se produce un contraste entre las características que considera negativas y que la inducen a justificar su acto de habla, y aquellos aspectos favorables, que validan su respuesta.

\subsection{Sobre los factores pragmático-discursivos: funciones de la atenuación}

En el género conversación coloquial, la justificación atenuante ocurre principalmente con la función de autoproteger la imagen del hablante y, en menor medida, se presentan casos en que los informantes buscan prevenir el daño a la imagen de su interlocutor y/o restablecer el consenso. El ejemplo (4) muestra la estrategia de autoprotección:

(4) B: [no/ si es como grave igual] porque $\mathrm{mm}$ como que le han salido como hilitos de sangre igual

$$
\mathrm{C} \text { : uu ¿y por qué no lo hai llevado? }
$$

B: porquee-porque al que lo llevo-es que he pensado dos cosas / llevarlo como a uno que está recomendado / pero no tiene su historial po y al que siempre lo llevo es terrible caro / pero tienen todo su historial // entonces- $§$ (AMERESCO_SCL_004_03_18).

Esta conversación gira en torno al perro de B que aparece en escena enfermo, situación que no pasa inadvertida para $\mathrm{C}$, quien le reprocha a su interlocutor no haberlo llevado al 
veterinario (“ ¿y por qué no lo hai llevado?”), pese a que el estado del perro es evaluado por su dueño como ("grave igual"). La pregunta de C desencadena la justificación de B, que corresponde a un acto asertivo de opinión realizado por medio de porque seguido de es que, donde se esbozan los motivos que le impiden consultar por la salud de la mascota. Considerando que la imagen del hablante como responsable del bienestar de su mascota está siendo amenazada, esta justificación funciona como estrategia de autoprotección, sobre todo considerando cuán expuesta ha quedado su imagen, ya que la evidencia, esto es, el mal estado de salud del perro, es visible para todos los interlocutores. En el ejemplo también se puede observar la manera en que la justificación opera solidariamente con la conjunción adversativa pero, la que le permite descartar las dos opciones de veterinario que dispone B, validando el hecho de no consultar por la salud del perro.

En el ejemplo que se presenta a continuación, se puede observar que las justificaciones funcionan tanto como estrategia de autoprotección como también de prevención de imagen de otro. Esta posibilidad de combinar más de una estrategia de imagen es algo que se genera en mayor medida en el corpus +/-formal de entrevistas, como se verá posteriormente en el ejemplo (6):

(5) B: y después llega y va para el cuarto que estábamos con tu papá / y dice / que «chao» / y yo llego y me di vuelta/ no lo pesqué // digna

A: orgullosa

B: (bueno / la huevada que sea)

A: [porque a mí me dolió po]

B: [es que S] / mira te voy a decir lo mismo que me dijo mi madre pero te lo voy a decir con una delicadeza extrema para que no te sno te marque a- por el resto de tu vida como me marcó a mí que me haya dicho mi madre /// tú / erí como yo // que llegamos y largamos / ¿cierto? ¿sí o no? / y como que no sabemos tener tino / a veces no queremos ofender ni lastimar a nadie $\uparrow / /$ pero con lo que decimos los lastimamos sin intención / ¿sí o no? (AMERESCO_SCL_008_03_18).

En el ejemplo (5), madre e hijo (B y A, respectivamente), comentan sobre algunos aspectos negativos del hermano de A, luego de que ambos hermanos tuvieran una pelea. La justificación de $\mathrm{B}$ introducida por es que se construye en un acto asertivo a través del cual 
la madre le señala a su hijo que no tiene "tino", es decir, que es una persona que emite juicios hacia terceros sin moderación. La justificación le permite a la madre autoproteger su imagen, pero principalmente persigue prevenir el daño a la imagen de su hijo frente a la crítica. Prueba de ello es la utilización de otros recursos lingüísticos que permiten atenuar la opinión, tales como el uso de "mira", la introducción de recursos valorativos ("delicadeza extrema"), el uso del nosotros inclusivo ("llegamos y largamos"), las comparaciones (“como que"), el uso de "a veces", y finalmente, las preguntas retóricas que abren el espacio a posturas alternativas (“¿ sí o no?”, “ ¿cierto?”).

Como se ha adelantado, en lo que respecta al corpus $+/$ - formal de entrevistas semidirigidas puede observase que en el total de los casos donde se presenta una justificación atenuante, los informantes tienen la intención de autoproteger su imagen y/o prevenir un conflicto con el otro con el que interactúan:

(6) E: ¿cuál es la diferencia entre los amigos que se tienen de niños y los que se tienen de adulto? // I: no le veo mucho la diferencia // E: mmm // I: no le veo mucho la diferencia/ porque son dos épocas de la vida tan distintas/ los amigos de niño / bueno / hay dos tipos de amigos de niño según yo / los amigos que son amigos porque los padres son amigos/ o porque las familias son amigas/ y los amigos que uno se hace en el colegio/ en eh/ en en las fiestas/ en eeh entonces son otros tipos de amigos que/ que siendo de la misma edad/ de la/ de la/ de la infancia/ o de la juventud/ son un poco distintos (PRESEEA_SCHI_M33_103).

En este ejemplo, pueden observarse dos de las tres funciones de la atenuación. Por un lado, la informante se autoprotege proporcionando un argumento sobre por qué cree que no hay diferencias y, por otro, con la inclusión de porque para introducir el argumento previene un conflicto con el otro, dando incluso opciones de lo que considera tipos de amigos: los amigos que provienen del ámbito familiar y los amigos que se hacen en la infancia o juventud. Habiendo construido un discurso con alternativas precedidas de una fórmula para acotar la opinión a la propia persona ("según yo") su opinión se restringe y evita así un conflicto con su interlocutor. En este ejemplo, es relevante además el empleo de "тисho" con una repetición, porque si bien esta partícula constituye un intensificador, opera como cuantificador atenuante para decir que no hay una gran diferencia. Son finalmente 
importantes el uso de la forma impersonal “uno”, que en este caso, además, cumpliría una función ejemplificadora, y el empleo del cuantificador "un poco".

En relación con los hallazgos expuestos, el estudio de De Andrade refuerza que los fenómenos de atenuación en actos asertivos se tienden a emplear para expresar cortesía para la valoración de la imagen de los hablantes. Los atenuantes, en consecuencia, indicarían una vacilación o un alejamiento de la aserción o bien sugerirían la apertura y la posibilidad de intercambiar opiniones o ideas. Esto ocurre mayormente, según se ha descrito en la investigación que presentamos, en el género conversación coloquial, donde la imagen de cooperación e igualdad entre los interlocutores es la que más se valora. En su trabajo, De Andrade también concluye que la atenuación asertiva en Chile constituye una estrategia que permite prevenir potenciales daños a la imagen propia basados en la evaluación de los interlocutores.

\subsection{Sobre los factores estructurales}

En el género conversación coloquial la gran mayoría de las justificaciones, además de generarse en los tipos textuales argumentativos, se presentan al inicio del turno de conversación, el que generalmente funciona como una reacción y/o interrupción frente al comentario, opinión o bien el reproche de otro interlocutor en el turno de conversación precedente. Esto se identifica como el discurso desencadenante en este contexto. Esta es una característica que diferencia claramente los géneros en estudio. Así, por medio de la justificación en inicio de turno el hablante no solamente busca justificar el desacuerdo provocado por un otro, sino que también persigue proteger su imagen potencialmente dañada debido a su interrupción.

(7) B: [pero es que] en el campo es como la celebración de la vida de la persona po no es tanto la pena por la muerte o sea así lo entiendo yo $\S$

$\mathrm{C}$ : $\quad \S$ es que estaban todos llorando y un rato después todos comiendo así como felices $\S$

B: $\S$ claro pero iguall/ llorai po

A: es que al final/ yo me daba cuenta que/l dentro del mismo funeral como quee tamp- el muerto igual pasaba a segundo plano en algún momento porque tú empezai a ver- a- a ver gente que no veíai 
hace mucho tiempo y como que a contar tu vida y la historia y no sé quél como o ¿te acordai de cuando habíamos salido con él? y blablablá empiezan como a revivir historias o a contar otra historia aparte entonces como que ya el funeral deja de estar y empieza como la otra parte ¿cachai? § (AMERESCO_SCL_003_03_18).

En este caso, se puede observar cómo los turnos de todos los interlocutores A, B y C comienzan con una justificación introducida por es que, la que funciona como mecanismo de interrupción al otro, además de las funciones anteriormente descritas de autoprotección y prevención de imagen.

En esta conversación en particular, los amigos discuten sobre los funerales y cómo estos se asemejan a verdaderas celebraciones sobre todo en el sur del país. Se trata de un tema que podría ser controversial, ya que A señaló en turnos conversacionales anteriores que no había llorado con la muerte de su abuelo, puesto que fue en el campo y el funeral se transformó en una celebración. La primera intervención de B busca el acuerdo con A, justificando los motivos por los cuales en el campo se celebra a los muertos. Por otra parte, $\mathrm{C}$ interrumpe a $\mathrm{B}$, justificando el desacuerdo $\mathrm{y}$ su interrupción por medio de es que. Finalmente, A interrumpe a B y construye un turno de conversación más extenso sobre una justificación que apunta a explicar por qué no lloró a su abuelo en el funeral. Esta justificación se acompaña de diversos recursos lingüísticos que permiten atenuar aún más su opinión, como el uso de “yo”, que restringe la opinión a su propia persona, permitiendo otros puntos de vista, el uso de comparaciones introducidas por "como que" y el marcador discursivo "igual”. Estos resultados coinciden con la investigación de España, quien señala que el "es que" en inicio de turno se utiliza con la función pragmática para auto-adjudicarse el turno de conversación, sin que ello sea visto como una infracción por el interlocutor. Por medio de esta justificación, el hablante protege su propia imagen, a la vez que la imagen negativa de su interlocutor.

En el género entrevista semidirigida el total de casos de justificaciones atenuantes aparece en segmentos argumentativos, del mismo modo que se da en la conversación coloquial. Estas actividades retóricas se generan por un desencadenante que es una pregunta directa o una solicitud de opinión en torno a ejes temáticos que representan en algún grado un tema 
controversial para los informantes, entre otros, el cambio climático, la política, la seguridad del barrio en el que vive y el sistema de transporte de la ciudad.

(8) E: eeh // si cree que el clima ha cambiado en Santiago los últimos // I: sí por ejemplo antiguamente / el mejor eeh / el mejor eeh mes para ir a veranear / para ir a veranear era eeh febrero ahora febrero está malo // se puso malo / es como marzo de antes / entonces ahora los mejores meses son diciembre y enero / pero febrero ya está ya está fría la costa / totalmente / antes uno se podía bañar pero ahora uno no se puede bañar prácticamente // cambió mucho el clima (PRESEEA_SCHI_H33_097).

En el ejemplo precedente, ante el requerimiento de opinar sobre el clima, el informante construye su argumento sobre la base de una comparación que le sirve como garantía para la argumentación. Para atenuar más todavía su aserción, incluye el uso reiterado de "uno" impersonal y del adverbio "prácticamente" que actúa como un modificador externo del significado. En el ejemplo (8), además, el hablante reduce el potencial daño a su imagen puesto que considera que existe la posibilidad de que su interlocutor no esté de acuerdo con lo que señala. En este sentido, la comparación funciona como un atenuante mediante el cual dos estados del mundo pueden contribuir a argumentar mejor su aseveración. La extensión del acto de habla le permite en paralelo reforzar un discurso no impositivo con otros atenuantes.

A partir de los hallazgos descritos, se podría decir que las estrategias de atenuación, especialmente desde la dimensión estructural, son empleadas de formas muy típicas en un determinado género, lo que estaría vinculado con el tipo de registro.

\subsection{Sobre los factores enunciativos}

En el corpus conversacional la gran mayoría de las justificaciones atenuantes ocurren en actos asertivos de opinión, como se ha mostrado en los ejemplos precedentes. Estas argumentaciones se desencadenan por alguna pregunta, opinión o comentario de otro interlocutor en un turno de conversación previo, que siempre suponen un riesgo a la imagen de los hablantes. A pesar de que se trata de escasas ocurrencias, es relevante mencionar que 
se observaron algunos casos de justificaciones en actos directivos y en actos expresivos, como muestran los ejemplos (9) y (10):

(9) C: ¿por qué no me ve allá un sobre?// veme un sobre $\uparrow /$ hay una carpeta dee la clínica/ necesito ver un papel/ o en las bolsas [no sé si llevé las bolsas]

$\mathrm{B}$ : [K acuéstate]

C: es- es unn sobre/ de I o de la clínica L- la clínica D/ porque tengo que ver un papel $\uparrow /$ es un sobre grande $\S$ (AMERESCO_SCL_007_03_18).

En este ejemplo, la intención comunicativa del acto de habla es solicitar que el interlocutor realice algo por el hablante, en este caso, que B le entregue un sobre con los resultados de exámenes a $\mathrm{C}$. Este acto de habla se calcula como riesgoso para la imagen tanto del hablante como del oyente, puesto que invade el territorio del otro, razón por la cual la solicitud se construye sobre la base de una justificación introducida por "porque”, que reduce la fuerza ilocutiva del acto y con ello las posibilidades de desacuerdo.

La justificación atenuante, como se mencionó anteriormente, también ocurre en actos de habla expresivos:

(10) A: ya a ver / perdón que estaba haciendo otra cosa / dícteme de nuevo / R como mi nombre ¿no? ere-o-y/ [¿o no?]

B: [mm] (AMERESCO_SCL_002_03_18).

En este caso, la intención ilocutiva del hablante consiste en expresar disculpas por no poner atención a su interlocutor, quien le solicita que busque el nombre de una figura pública en internet. Como la disculpa pone en riesgo la imagen del hablante, este ofrece una justificación por medio de que para autoproteger y minimizar los costos a su imagen y también prevenir daños a la imagen de su interlocutor por haber sido ignorado.

En la entrevista semidirigida, por su parte, los elementos que definen al acto de habla como tal se asocian con el desencadenante de la justificación. A diferencia de lo que ocurre en el género conversación, en este caso los hablantes siempre construyen actos de habla asertivos, de manera que se comprometen con la verdad de la proposición expresada. 
(11) E: ¿ha habido cambios en el barrio en todo este tiempo que ha estado viviendo ahí? // I: ¿cambios? // E: sí // I: sí porque el alcalde que es el señor $\mathrm{P}$ que fue reelegido otra vez como la quinta vez que es reelegido / se nota que trabaja bien porque esta todo limpio eeh / las calles limpiecitas // parques bonitos con fuente / los semáforos donde corresponden / no hay hoyos en la calle // se nota que el hombre trabaja / se preocupa / por eso que fue reelegido como por cuarta quinta vez // ya no interesa partido si no que el hombre trabaja muy bien (PRESEEA_SCHI_H33_097).

En el ejemplo anterior, la fuerza ilocutiva del acto de habla consiste en que el hablante afirme algo, con diferente nivel de certeza. Se trata evidentemente de un tema controversial, puesto que incluye un componente político, de manera que primero se incluye una justificación introducida por "porque" con la que el hablante se autoprotege, y luego aparece una explicación, a la que se llega luego de presentar los argumentos y se introduce mediante "por eso que". Con esta última parte de su intervención, el hablante previene un conflicto con el otro al que se dirige, en especial porque, hacia el final, señala que no es un asunto de partidos políticos, sino de trabajo efectivo.

En relación con los hallazgos expuestos, Breñes Peña destaca que los actos asertivos pueden constituirse como intervenciones reactivas o reactivo-iniciativas en el par adyacente aserción-respuesta, que pueden indicar no aceptación o rechazo parcial o total sobre lo dicho. Así, su grado de fuerza ilocutiva es mayor a la de un acto de aceptación. Este tipo de actos, siguiendo al autor, presentan rechazo de lo dicho, rectificación de lo negado y justificación del desacuerdo. Sobre este último aspecto es que se construyen los actos de habla que forman parte del estudio propuesto y donde sería necesario el empleo de atenuantes que justifiquen aquello que se expresa.

\subsection{Sobre los factores situacionales}

En la última de las dimensiones abordadas, debe precisarse que en el género +/- formal, conformado por la entrevista semidirigida, se registran varias diferencias en relación con el género +/- coloquial de la conversación. El registro en la conversación coloquial se 
caracteriza por ser informal y por tener un menor nivel de planificación, lo que se expresa en turnos de conversación dinámicos, con variadas interrupciones y reformulaciones y también por un alto nivel de giros temáticos que aportan a los fines interpersonales de la comunicación. Como se ha mencionado anteriormente, este dinamismo de la conversación es la que permite que las justificaciones al inicio de los turnos de conversación sean tan productivas en este género. Como los hablantes tienen turnos más bien acotados, las justificaciones siempre se presentan como la reacción a un otro en un turno de conversación precedente, a diferencia de lo que ocurre en el género entrevista donde el hablante siempre justifica su propia opinión, en ambos casos con el propósito de proteger su imagen y/o la de su interlocutor.

A nivel de interlocutores, este corpus de conversación coloquial se compone por hablantes con relaciones más bien simétricas, tales como amigos, compañeros de hogar, compañeros de curso y familiares cercanos. Como se trata de personas que comparten a diario y tienen un vínculo emocional, tienen un gran número de temas cotidianos disponibles para discutir, los que también incluyen temas de opinión más controversiales, como la política, problemas familiares, y también temas más cotidianos como las comidas favoritas, el chisme y los partidos de fútbol, entre otros. Esta diversidad temática, sumada a la inmediatez comunicativa presente en la conversación coloquial, es la que permite actos de habla distintos al asertivo, tales como los casos pesquisados de actos directivos y expresivos.

La entrevista exige un mayor grado de conciencia en la medida en que deben establecer relaciones lógicas. Por ejemplo, la justificación como tal implica que los informantes presenten una opinión seguida de argumentos que la respalden, mientras que en la explicación se presentan primero los argumentos y luego la conclusión. Si bien no se usa un registro formal, como el que se podría esperar, ente otros, en el discurso académico, en este caso la formalidad está dada por el orden de las palabras y por la elección de una estrategia $\mathrm{u}$ otra para responder al desencadenante del acto de habla atenuado. En definitiva, el registro se caracteriza por su menor grado de planificación sobre la marcha, su menor fin interpersonal y su menor tono informal. Además, de acuerdo con Briz El español coloquial este registro tiene una menor relación de igualdad, donde los roles de entrevistador e informante están bien delimitados; una menor relación vivencial, puesto que no 
necesariamente habría un vínculo entre entrevistador y entrevistado; y un menor marco de interacción cotidiano y cotidianidad temática. Sobre esto último, como ya se ha mencionado, la temática varía, aunque siempre se organiza en torno a temas que podrían generar conflictos de opinión (cambio climático, sistema de transporte público, política, etc.).

Es interesante que los hallazgos anteriormente descritos se vean reforzados con las propuestas de otros investigadores. De Cock et al. precisan que en los géneros donde existe una relación asimétrica -en su caso, clases y entrevistas- la persona cuyo rol es más débil tiende a proteger su imagen propia mediante el uso de estrategias atenuantes, de modo que se contribuye al equilibrio de la relación existente entre los participantes en función del contrato conversacional que exige colaborar. Por su parte, en los géneros de relación simétrica -en su caso, debates y conversaciones-, el uso de atenuantes es más equilibrado entre los distintos participantes, lo que sería un reflejo del equilibrio que ya existe en su relación.

\section{Conclusiones}

Las principales conclusiones de esta investigación pueden sintetizarse de la siguiente manera:

Sobre los factores lingüísticos: en ambos géneros se observa que la estrategia lingüística mayormente utilizada es la justificación propiamente tal, seguida por la explicación y, en menor grado, las comparaciones. En la conversación, las justificaciones muestran variadas combinaciones y herramientas lingüísticas, mientras que en la entrevista semidirigida las combinaciones lingüísticas se restringen. Esto podría ser el resultado de la mayor formalidad y el menor dinamismo de este género. En las explicaciones los recursos lingüísticos suelen ser los mismos en ambos géneros del mismo modo que ocurre en las comparaciones. El análisis permite concluir también que es en el género entrevista semidirigida donde existe mayormente la posibilidad de utilizar más de una estrategia para 
justificar un mismo acto de habla, lo que podría deberse a que los turnos son menos dinámicos y más extensos.

Sobre los factores pragmático-discursivos: el estudio comparativo permite concluir que, en ambos géneros en estudio, los hablantes emplean estas estrategias para autoproteger su propia imagen y/o para prevenir un conflicto con su interlocutor. Es relevante también mencionar que los hablantes incluyen una serie de recursos de atenuación que operan de manera solidaria para lograr sus objetivos comunicativos, y que estos recursos se acompañan de términos valorativos, especialmente en el género conversación coloquial.

Sobre los factores estructurales: en la conversación coloquial las justificaciones tienden a presentarse al inicio del turno de conversación. La justificación opera aquí como una reacción y/o interrupción frente a un comentario, opinión o reproche de otro interlocutor en el turno de habla anterior. Esta es una característica que diferencia claramente los géneros en estudio, ya que en el género entrevista semidirigida las justificaciones se generan por un desencadenante en forma de una pregunta directa o una solicitud de opinión respecto de ejes temáticos que representan en cierta medida un tema controversial para los informantes. En ambos casos se trata de actos de habla que se insertan en secuencias argumentativas. Podría sugerirse entonces que las estrategias de atenuación se utilizan de formas muy típicas en un género determinado en función del tipo de registro.

Sobre los factores enunciativos: en el género conversación coloquial, las justificaciones atenuantes ocurren en general en actos asertivos de opinión, aunque se observaron algunos casos de justificaciones en actos directivos y en actos expresivos. En la entrevista semidirigida los atenuantes que justifican siempre se usan en actos de habla asertivos, en el sentido de que los hablantes se comprometen con la verdad de la proposición expresada.

Sobre los factores situacionales: en la conversación coloquial los turnos de conversación son dinámicos, con variadas interrupciones y reformulaciones, lo que permite que las justificaciones al inicio de los turnos de conversación sean tan productivas en este género, a diferencia de lo que ocurre en el género entrevista semidirigida, donde el hablante siempre justifica su propia opinión a partir de un desencadenante que suele ser una pregunta sobre un tema específico. Asimismo, la diversidad temática, sumada a la inmediatez 
comunicativa de la conversación coloquial, da la posibilidad de incluir actos de habla distintos al asertivo, tales como directivos y expresivos. Se trata en definitiva de géneros diferenciados por la menor relación de igualdad, la menor relación vivencial, el menor marco de interacción cotidiano y la menor cotidianidad temática. Estas características, como se ha descrito en la investigación propuesta, permiten observar diferencias y semejanzas en el empleo de la justificación atenuante. En este orden, queda pendiente la indagación sobre si existen diferencias específicas entre justificaciones, comparaciones y explicaciones entre los géneros en estudio.

\section{Bibliografía}

Albelda, Marta. “¿Cómo se reconoce la atenuación? Una aproximación metodológica basada en el español peninsular hablado”. (Des)cortesía en español. Espacios teóricos y metodológicos para su estudio (2010): 41-70.

Albelda, Marta. "Sobre la incidencia de la imagen en la atenuación pragmática". Revista Internacional de Lingüística Iberoamericana 14.1. (2016): 19-32.

Albeda, Marta. "Estrategias atenuantes en géneros discursivos del español”. Spanish in context 15. 2. (2018). Número monográfico.

Albelda, Marta y Cestero, Ana. "De nuevo, sobre los procedimientos de atenuación lingüística”. Español Actual 96 (2011): 121-155.

Albelda, Marta y Cestero, Ana. "Estudio de variación en el uso de atenuación I: Hacia una descripción de patrones dialectales y sociopragmáticos de la atenuación en español". Revista Signos (en prensa).

Albelda, Marta, Briz, Antonio, Cestero, Ana Kotwica, Dorota y Villalba, Cristina. "Ficha metodológica para el análisis pragmático de la atenuación en corpus discursivos del español (ES.POR.ATENUACIÓN)"'. Oralia 17 (2014): 7-62.

Bouzas, Paula. "Atenuación en textos informativos de prensa”. Normas 7. 2. (2017): 91110.

Briz, Antonio. El español coloquial en la conversación: esbozo de pragmagramática. Barcelona: Ariel, 2001.

Briz, Antonio y Grupo Val.Es.Co. Corpus de conversaciones coloquiales. Madrid: Arco Libros, 2002. 
Briz, Antonio y Albelda, Marta. "Una propuesta teórica y metodológica para el análisis de la atenuación lingüística en español y portugués. La base de un proyecto en común (ES.POR.ATENUACIÓN)". Onomázein 27. 2. (2013): 288-319.

Brenes Peña, Ester. Actos del habla disentivos. Identificación y análisis. Sevilla: Alfar, 2011.

Cestero, Ana. "Recursos lingüísticos de atenuación en el habla de Madrid estudio sociopragmático". Cum corde et in nova grammatica: estudios ofrecidos a Guillermo Rojo, eds. Tomás Jiménez, Belén López, Victoria Vázquez y Alexandre Veiga. Universidad de Santiago de Compostela, 2012: 233-246.

Cestero, Ana y Albelda, Marta. (2012). "La atenuación linguiística como fenómeno variable". Oralia 15 (2012): 77-124.

Contreras Fernández, Josefa. “¿Hay diferencia en las estrategias de atenuación en los correos electrónicos españoles y alemanes?" Miradas multidisciplinares a los fenómenos de cortesía y descortesía en el mundo hispánico, eds. Julio Escamilla Morales y Henry Vega. Barranquilla: Universidad del Atlántico-Programa EDICE, 2012: 451-471.

De Andrade, Adriana. "Actos asertivos y cortesía: las diferentes estrategias utilizadas por brasileños y chilenos en el contexto académico". Textos en Proceso 2.1. (2016): 25-53.

De Cock, Bárbara, Marsily, Aurelie, Pizarro Pedraza, Andrea y Rasson, Marie. “¿Quién atenúa y cuándo en español?”. Spanish in Context 15.2. (2018): 305-324.

España, Margarita. “Aspectos semántico-pragmáticos de la construcción «es que» en español”. DICENDA. Cuadernos de Filología Hispánica 14. (1996): 129-147.

Estrada, Olga y Zárate, Griselda. "Atenuación, género e intensificación en el discurso político: las reacciones mediáticas del caso de una legisladora de Nuevo León, México". Normas 2 (2015): 124-137.

Figueras, Carolina. (2018). "Atenuación, género discursive e imagen". Spanish in context, 15. 2. (2018): 258-280.

Flores, Elizabeth y Ramírez, Gaspar. (2015). "La atenuación de los actos asertivos: diferencias entre hombres y mujeres”. Sociocultural Pragmatics 3.1. (2015): 90-119.

Goffman, Erving. Interaction ritual: essays on face-to-face behavior. Nueva York: Anchor Books, 1967.

González García, Virginia y García Ramón, Amparo. "Atenuación e intensificación: estrategias pragmáticas del rechazo en respuestas a invitaciones en redes sociales en línea". Atenuación e intensificación en géneros discursivos, eds. Marta Albelda y Wiltrud Mihatsch. Madrid/Frankfurt: Iberoamericana/Vervuert, 2017: 187-204. 
González Riffo, Javier. Estrategias de atenuación en narraciones de experiencia personal de hablantes de Santiago de Chile: un estudio sociopragmático. Tesis para optar al grado de Magíster en Lingüística. Santiago: Universidad de Chile, 2017.

González Riffo, Javier. "Diminutivos atenuadores en narraciones de experiencia personal de hablantes de Santiago de Chile: ¿fenómeno variable?”. Cuadernos de Lingüística del Colegio de México 6.1. (2019): 1-31.

González Riffo, Javier y Guerrero González, Silvana. "Estudio sociolingüístico del empleo de gradación de valoraciones inscritas en discursos argumentativos en el corpus PRESEEA de Santiago de Chile". Linred 14 (2016). http://www.linred.es/articulos_pdf/LR-articulo10102016.pdf

González Riffo, Javier y Guerrero González, Silvana. "Estrategias de atenuación en narraciones conversacionales”. Lengua y Habla 21 (2017): 29-44.

González Riffo, Javier y Guerrero González, Silvana. "Recursos de atenuación en la 'orientación' de narrativas personales orales desde una perspectiva sociopragmática". Nueva revista del Pacífico 68 (2018): 62-81.

Guerrero, Silvana. "La atenuación lingüística en el corpus PRESEEA de Santiago de Chile". Alpha (en prensa).

Guerrero, Silvana, Gajardo, Consuelo, González, Javier y Reyes, Antonia “"“Lo que pasa es que la política se ha puesto farandulera": justificaciones atenuantes de aserciones de opinión en el corpus PRESEEA de Santiago de Chile". Literatura y Lingüística (en prensa).

Guerrero González, Silvana y González Riffo, Javier (en línea). "Corpus de conversaciones Ameresco-Santiago de Chile". Corpus Ameresco, www.corpusameresco.com, Coords. Albelda, Marta y María Estellés. Valencia: Universitat de València, ISSN: 2659-8337.

Helfrich, Uta y Pano, Ana. "Introducción: la atenuación en los discursos digitales en español”. Círculo de Lingüística Aplicada a la Comunicación 73 (2018): 5-14.

Infante, José María y Flores, María Eugenia. "Una aproximación a la atenuación y sus recursos en el discurso de políticos mexicanos Those who say that yes without saying yes". Normas 7.2. (2017): 72-90.

Meyer-Hermann, Reinhard. "Atenuación e intensificación (análisis pragmático de sus formas y funciones en el español hablado". Anuario de Estudios Filológicos 11 (1988): 275-290.

Montecino, Lesmer. "Cortesía y modalización en un foro de opinión en internet". Onomázein 8 (2003): 73-98. 
Montecino, Lesmer. "Intensificadores en el habla coloquial de jóvenes chilenos". Onomázein 9 (2004): 9-32.

Moya, Patricio y Carrió-Pastor, María. "La atenuación en los comentarios sobre las noticias digitales en periódicos de España y Chile”. Onomázein 40 (2018): 56-76.

PRESEEA. Corpus del Proyecto para el estudio sociolingüístico del español de España y de América. Alcalá de Henares: Universidad de Alcalá (2014-) http://preseea.linguas.net

Puga, Juana. La atenuación en el castellano de Chile. Un enfoque pragmalingüístico. Valencia: Universidad de Valencia, 1997.

Villalba, Cristina. "Atenuación: algunas claves metodológicas para su análisis". Normas 8.1. (2018): 306-316. 